Письма в ЭЧАЯ. 2009. Т. 6, № 2(151). С. 285-289

\title{
COMPUTER SIMULATION OF SPECTROMETER MAGNETS FOR SOME EXPERIMENTAL INSTALLATIONS
}

\author{
E. P. Zhidkov, R. V. Poljakova, I. G. Voloshina, E. E. Perepelkin, \\ N. S. Rossiyskaya, T. V. Shavrina, I. P. Yudin \\ Joint Institute for Nuclear Research, Dubna
}

The significance of numerical simulation in the research of magnetic systems is determined by not only known advantages of the computing experiment, but also by the fact that the measurement of a magnetic field is a labour-consuming and expensive problem. Mathematical simulation allows one to investigate those parts of the magnet's design where the measurements of the magnetic field are extremely complicated or even impossible.

This work is aimed to generalize experience of the mathematical simulation of magnetic systems of various-type physical and electromechanical installations and to work out some recommendations of the optimal use of some software products for the numerical modeling of magnetostatic problems. This work also presents some results of a numerical analysis of the magnetic systems of the JINR's physical installation MARUSYA with the purpose of studying an opportunity of designing magnetic systems with predetermined characteristics of the magnetic field.

Значение численного моделирования при исследовании магнитных систем определяется не только известными достоинствами вычислительного эксперимента, но и тем, что измерение магнитного поля является трудоемкой и дорогостоящей проблемой. Математическое моделирование дает также возможность исследовать те части конструкции магнита, измерения магнитного поля в которых или крайне затруднены, или даже невозможны.

В настоящей работе предпринята попытка обобщить опыт математического моделирования магнитных систем различного типа физических и электромеханических установок и дать некоторые рекомендации оптимального использования некоторых программных продуктов для численного моделирования магнитостатических задач. В работе также представлены результаты численного анализа магнитной системы физической установки МАРУСЯ ОИЯИ с целью изучения возможности конструирования магнитной системы с заданными характеристиками магнитного поля.

PACS: 02.60.Lj; 29.30.Aj

\section{INTRODUCTION}

The magnetic systems are present in Nature at various levels: these are magnets of experimental physical installations, electromotors, etc. Nowadays one of the basic methods used for designing and creating magnetic systems is a mathematical simulation [1].

The computer modelling of a magnetic system means construction of a mathematical model that describes it, creation of a numerical algorithm and its realization in the form of a software complex on a computer with inclusion in it of a system of graphic software for color 
and usual one-colour visualization of obtained numerical results, solutions with wide use of service convenient for work, for example, with the help of databases and specialized shells in the form of systems based on knowledge, i.e., expert systems. The computer modelling enables one to reduce sharply the time of analyzing the field in the magnet of selected configuration, to increase accuracy and to reduce the cost of both the analysis and the magnet; i.e., the mathematical modelling of magnetic systems actually is the tool that allows one numerically to make a choice of the optimum magnetic system in each particular case.

The process of mathematical simulation of magnetic systems should be divided into two big stages. At the first stage of designing a new magnetic system, one needs to have an opportunity of its fast and operative modeling with the help of software that possesses the properties of a «slide rule», namely, availability, simplicity of use and sufficient accuracy of numerical computations.

At the second stage, the configuration of the magnetic system, chosen as a basis, should be studied in more detail; i.e., it is necessary to perform more exact numerical calculus in both two-dimensional and three-dimensional cases. For a detailed research on the properties of the magnetic systems in the two- and three-dimensional cases, one should apply optimal numerical algorithms and their realization as software products.

This work presents experience of the mathematical simulation of magnetic systems of various-type physical and electromechanical installations and gives some recommendations of optimal use of some software products for numerical modeling of magnetostatic problems.

\section{SOFTWARE SUPPORT OF SOLVING A MAGNETOSTATIC PROBLEM}

Work [2] gives a detailed description of numerous numerical methods and accordingly software products for all stages of solving magnetostatic problems, namely, the entry block (preprocessor: description of entrance data), the block of construction of an accounting lattice, the block of construction of discredited equations, the block of solving a system of algebraic equations (processor), the block of processing results and their visualization (postprocessor).

Giving due to specialized software packages for solving magnetostatic problems of a certain type, it is necessary to note quite a justified aspiration of developers of magnetic systems to possess a tool of mathematical simulation of magnetic systems simple in use and reliable enough in sense of obtained numerical results in both two-dimensional and threedimensional cases.

I. For solving magnetostatic problems in a two-dimentional case, a software complex KPMMS has been designed for numerical modelling of magnetic systems, which is based on the software complex POISSON.

The KPMMS software complex includes:

1) A program of intellectual support (expert system) of making decision for optimal choice, for example, of a configuration of the magnetic system.

2) A program of polynomial representation of components of the magnetic field with the purpose of their further use in problems of charged particles dynamics.

3) A program COBRA-M - numerical modelling of some class of nonlinear inverse problems of magnetostatics for mathematical simulation of iron-free magnetic systems with a rectangular aperture [3]. 
II. For solving magnetostatic problems in a three-dimensional case, a software complex [4] of numerical modelling of magnetic systems for two-scalar potentials has been created. An algorithm of making numerical calculations more precise is included in this software complex for the problems containing the so-called «angular points».

When computing particular magnetic systems, for whatever reasons it was necessary to conduct some additional studies on the accuracy of the obtained solution and thus to use additional numerical methods to make the solution to the magnetostatic problem more precise, namely:

1) Research on the influence of putting the external border $\Gamma$ of a boundary-value problem on the accuracy of fulfillment of the boundary condition $u(\infty)=0$. It has been found numerically that the putting of the external border not strongly influences the accuracy of numerical calculations, which allows one to put border $\Gamma$ close to the location of the magnetic system and enables one «to save» points of the difference scheme by throwing all of them all over the working area of the magnetic system.

2) It should be noted that during the numerical modeling of some magnetic systems, an additional control over the accuracy of approximation of condition $u(\infty)=0$ over Richardson [5] is performed.

3) When calculating a particular magnetic system, the area in which the boundary problem is solved, often has everywhere a smooth border, excepting the final number of angular points in the vicinity of which the border is formed by crossing two smooth curves. In such cases the solution to the problem or derivative solutions can have a special feature. In [4] the question of behaviour of the magnetic field in vicinities of «angular points» is studied by means of algorithms which give the best accuracy of solving the problems of magnetostatics with rectangular aperture. At numerical calculation such a method gives the results which are compatible in accuracy with the results obtained on lattices with 4-5 times greater number of units along each axis.

\section{RESULTS OF THE NUMERICAL SIMULATION OF THE MAGNETIC SYSTEM OF INSTALLATION MARUSYA (VBLHE, JINR)}

Work [6] presents the results of mathematical simulation and comparison of a computer distribution of the magnetic field with the conducted measurements of the field of the modified magnet SP-40 of installation MARUSYA (VBLHE, JINR). As an example, this work shows part of the results of numerical simulation of the above-mentioned magnetic system. Figure shows the dependences $B_{y}(x, 0, z), B_{y}(x, 0.05 m, z), B_{x}(x, 0.05 m, z), B_{z}(x, 0.05 m, z)$ for 600 A current. Figure $a$ gives a distribution of the basic component of field $B_{y}(x, 0, z)$ on the median planes $(y=0)$. The area of a homogeneous field at the level $1.21 \mathrm{~T}$ is located entirely under the magnet's pole falling down on the edges of the pole (both in a cross direction and on the beam) up to sizes of the order of $8 \mathrm{G}$ for $z=2.50 \mathrm{~m}(x=y=0)$ and up to $0 \mathrm{G}$ in a cross direction for $x=1.35 \mathrm{~m}(y=z=0)$. Figure $b$ shows a distribution of the basic component of field $B_{y}(x, 0.05 \mathrm{~m}, z)$ on plane $y=0.05 \mathrm{~m}$. The area of the homogeneous field at the level of $1.21 \mathrm{~T}$ is also located under the magnet pole. Further the field also falls down at the edges of the pole (both in a cross direction and on the beam) up to sizes of the order of $5 \mathrm{G}$ for $z=2.50 \mathrm{~m}(x=y=0)$ and up to 0 in a cross direction for $x=1.35 m(y=z=0)$. Figures $c$ and $d$ give distribution of cross and longitudinal 

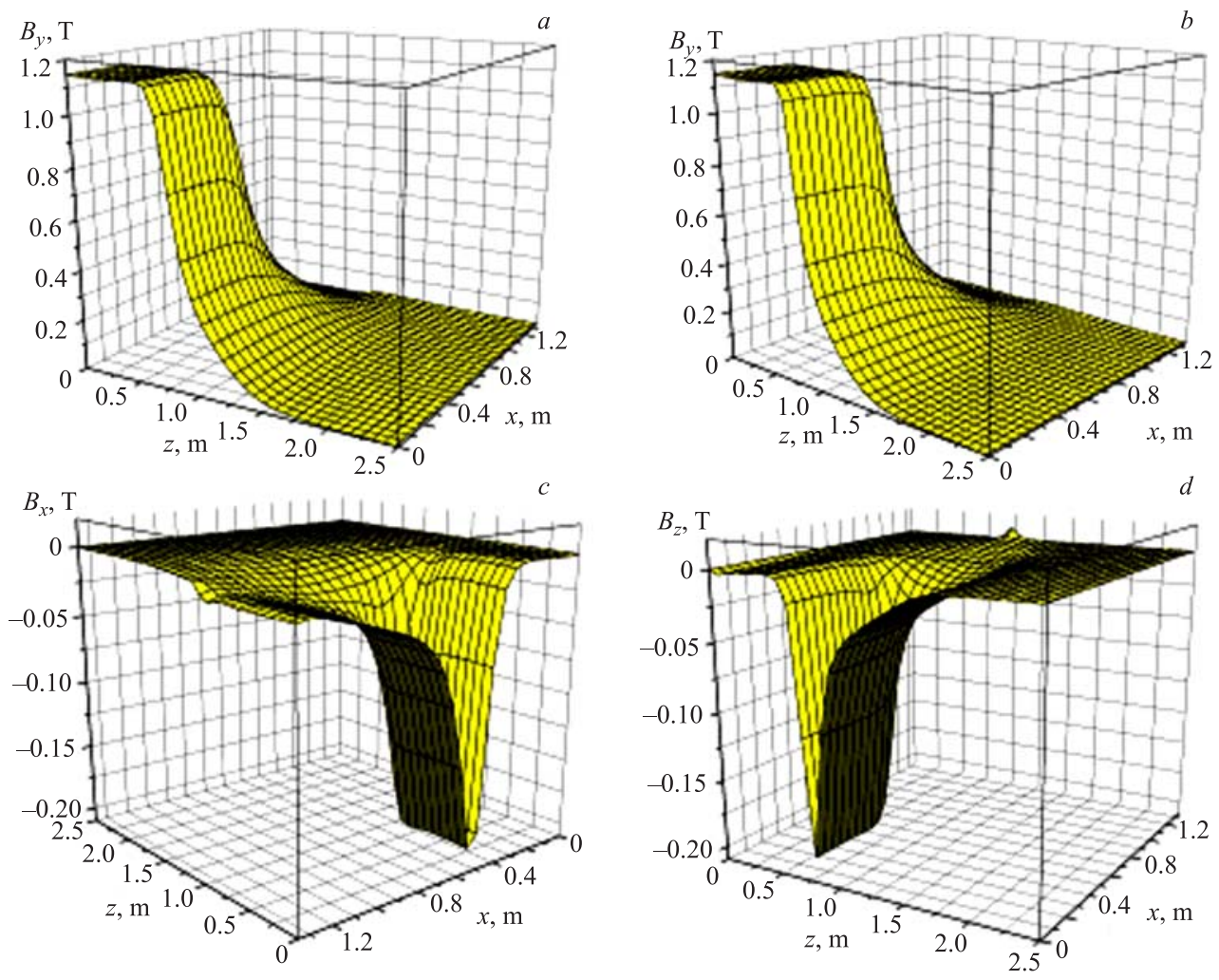

Spatial distribution of $B_{x}, B_{y}, B_{z}$ magnetic field SP-40 for $I=600$ A

components of field $B_{x}(x, 0.05 \mathrm{~m}, z)$ and $B_{z}(x, 0.05 \mathrm{~m}, z)$ on plane $y=0.05 \mathrm{~m}$. As shown in figure, the proposed procedure of numerical modelling of the magnetic system under consideration adequately describes the nonlinear measurements of the form of the magnetic field depending on the current in winding.

The obtained numerical results are used for carrying out the computer modeling of the MARUSYA installation and experiment on this installation and, after carrying out physical data taking sessions, are used for processing those data.

\section{CONCLUSION}

1. The KPMMS software complex has been created which allows one to solve a wide class of magnetostatic problems in a two-dimentional case in the cartesian and cylindrical systems of coordinates. It also allows one to accumulate a databank of numerical models of magnetic systems, enabling one to facilitate and accelerate considerably the process of creation and reconstruction of magnetic installations. A software complex for calculation of 3D distribution of a field of magnetic systems has been developed.

2. Numerous calculations of magnetic systems and comparison of numerical calculations with experimental data have shown that the computer distribution of the magnetic field in 
most cases with $1 \%$ accuracy (or less) coincides with experimental data. It seems sufficient for carrying out a numerical research on the influence of various parameters of the magnetic system on the size and the form of the magnetic field.

3. With the help of the suggested procedure, a lot of important practical problems have been solved the results of which have been put as a basis for reconstruction and creation of magnetic systems of installations [6,7].

Acknowledgements. The authors would like to thank a lot Professor A. A. Baldin for physical problem formulation.

\section{REFERENCES}

1. Samarsky A.A., Mikhajlov A.P. Mathematical Modelling. Ideas. Methods. Examples. M.: Nauka, Fizmatlit, 1997.

2. Ayrjan E. A. et al. Numerical Algorithms of Calculating the Magnetic Systems of Charged Particles Accelerators // Part. Nucl. 1990. V.21, No.1. P.251-307.

3. Polyakova R. V., Zhidkov E.P., Yudin I.P. Regularization by A. N. Tikhonov in One Problem of Magnetostatics // Proc. of the 13th Conf. «Mathematics. Computer. Education». Dubna, 2006.

4. Yudin I. P., Perepelkin E. E. 3D-Field Calculations of Magnets by the Two Scalar Potential Method. Report No.P-084 at ICAP-2004, St. Petersburg, 2004.

5. Zhidkov E. P. et al. Preprint No. 187. Dept. Comp. Math. Acad. of Sci. of USSR. 1988.

6. Baldin A. A., Yudin I. P. Numerical Modelling of Distribution of the Magnetic Field of Magnet SP-40 of Installation MARUSYA and Comparison of Results with Experimental Data. JINR Preprint P11-2006-99. Dubna, 2006.

7. Polyakova R. V. et al. Calculation of Distribution of the Spectrometer Magnet Field // Part. Nucl., Lett. 2006. V.3, No.7(136). P.74-77 (Proc. of the Conf. Devoted to the Memory of Prof. V.P. Sarantsev, Alushta, Sept. 8-10, 2005).

Received on February 14, 2008. 